\title{
Investigation of geometric parameters' influences on the measurement uncertainty by various measuring sensors
}

\author{
Rauf Oezden* and Marcel Sabin Popa \\ Technical University of Cluj-Napoca, Department for Manufacturing Technology, Romania
}

\begin{abstract}
The following paper was written in cooperation with the TU of Cluj-Napoca and provides an overview on the possibilities of calculating measurement uncertainty in the industrial environment of geometrical measurement. Scheduling effective influences reveals measurement uncertainties. Furthermore, experimental approaches, which prove that measurements with different sensors arrive at difficult to reproduce data, are presented. Experimental methods, which reveal inevitable errors in measurement because they violate the laws of mathematics, are presented as well.
\end{abstract}

\section{Introduction}

Geometrical measurement is an integral part of the industry. Component evaluation and process control require great reliability on the part of the measurement results.

Often there is no clear statement on a measurement's accuracy and the uncertainties it is fraught with. Reason for this is commonly poor knowledge of the origin of measurement uncertainty of the influences that affect the results and their scope. Furthermore, there is a lack of experience on how to deal with individual influence factors.

Today, a measurement result, which was determined in a single measurement, is often accepted as the true value of a test object, but the measurement result is always an estimator, which is synthesized from several measurements. The true value of an item is imperfect and not accurately determinable in its nature. Each measurement is subject to influences, either by the environment, in which it is conducted, by the gauge, by the operator, by the measurement strategy or by the type of measurement subject.

Measurement uncertainty is considered to be a quantitative dimension for the quality of the respective measurement result. It is associated with the measurement result and characterizes the area of values, which can be ascribed to the measurement parameter through the conducted measurement. It answers the question of how well the determined result mirrors the measurement parameter's true value. Furthermore, it facilitates the assessment of measurement results' reliability, enabling comparisons of other measurements with the same measurement parameter or comparisons with reference values. Trust in the comparability of measurement results is very important in international trade. It

*Corresponding author: rauf.oezden@outlook.com 
helps to remove trade barriers. Since production engineering constantly evolves, driven by international competition, tolerances decliner more and more. Components become increasingly smaller and delicate. Production measurement technology settles immediately in or close to production. Correspondingly, the requirements for gauges in terms of speed, accuracy, reliability and sensitivity permanently increase. Thus, the effort to determine reliable measurement results increases. In order to understand and adapt properties such as influences, which affect measurement results, the calculation of measurement uncertainties through modeling or the assessment of causes, it is crucial consider the standards of product specification. Standards guarantee for a consistent approach in reducing inaccurate assessments and in creating global comparability of results. The particular advantage is the international confirmation of standards.

\section{Measurement errors and measurement uncertainty}

Measurement results can only comparable when they have been obtained under reproducible conditions. Measurement results shall become comprehensible through the designation of reference conditions [1]. Among the reference conditions is the reference temperature, which is set to $20^{\circ} \mathrm{C}$ in accordance with the standard [2].

Furthermore, the complete measurement sequence (measurement under repeatability conditions or under comparability conditions) and the influential variables, which themselves are not part of the measured variables, but influence the measured value, have to be constituted. In the measurement of geometrical quantities, the temperature of the measured object and its environment are important influential variables, which can entail measurement deviations.

The measuring sensors' user strives to keep measurement deviations to a minimum with the help of suitable measurement strategies, favorable measurement sequences and shielding from disturbances.

Figure 1 illustrates the path via the measurement deviations towards the measurement results and the occurring measurement uncertainties.

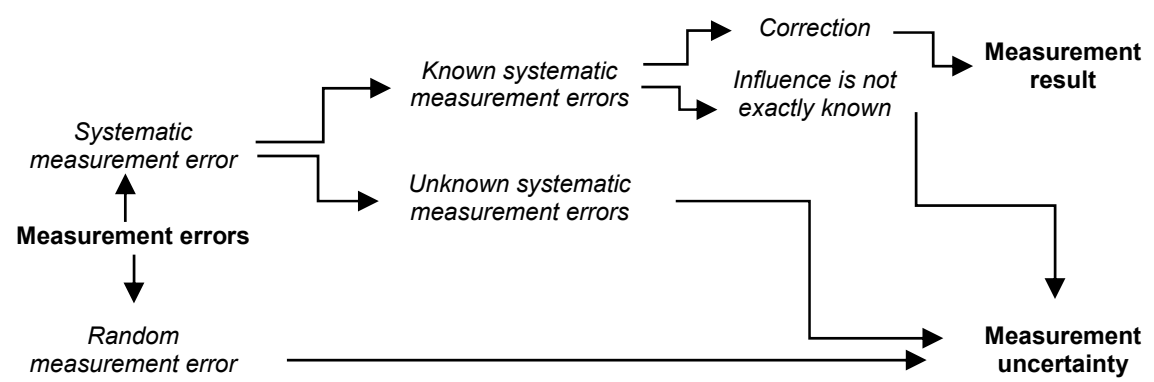

Fig. 1. Influences that describe a complete measurement result.

As far as the systematic measurement deviations are determined under comparability conditions, they are considered to be known and can influence the measurement result als corrections. The absolute value of the correction complies with the systematic measurement deviation, although with opposite portents.

Random measurement deviations can only partially be estimated via measurement sequences under repeatability conditions, because they still contain unknown fractions of the systematic measurement deviations. It is not known here, with which portent the measurement uncertainty is afflicted [1]. Therefore, it is included in the measurement result as portent undetermined value $( \pm)$. 


\subsection{Determination of measurement uncertainty}

\subsubsection{Fundamentals}

The definition of measurement uncertainty renders it a quantitative measure of the respective measurement result's quality. It answers the question of how well the obtained result mirrors the measurement's value. It enables the user to evaluate the reliability of the measurement result, e.g. to compare the results of different measurements with the same measurement values to each other or to reference values.

Often, a measurement value is to be compared to threshold values, which are defined in a specification or normative provision. In this case, the measurement uncertainty can yield whether the measurement result is clearly within the predefined limits or whether it hardly meets the demands. When the measured value is close to a limit value, there is a high risk for the measurement value not to meet the required demands.

\subsubsection{Important policies}

An overview of relevant standards and policies for the determination of measurement uncertainty in geometrical measurement technology is listed in the following:

- DIN EN ISO 14253 Inspection by measurement of workpieces and measuring equipment

- DIN EN ISO/IEC 17025 Requirements for the competence of testing and calibration laboratories

- DIN V ENV 13005 Guide to the Expression of Uncertainty in Measurement (GUM)

- VDA5/ISO22514-7 Test suitability of measurement/Capability of measurement processes

- VDI/VDE 2617 Accuracy of coordinate measuring machines

Today's high-tech economy with worldwide quality standards demands safe and particularly precise measurements. Only thus, extensive division of labor, precision fit, long life, product and consumer protection are possible. The basis for this are international standards and the tracing of any measurement values with known measurement uncertainty. An internationally accepted approach to measurement uncertainty determination is provided in the GUM (Guide to the Expression of Uncertainty in Measurement). In order to apply this comprehensive guide in accordance with the standard, a simple procedure for the determination of measurement uncertainty on the basis of GUM is developed in the international standardization committees [3]. In the process, a cost-optimized solution is developed for a certain measurement problem via an iterative method in consideration of measurement uncertainty. This approach is called the PUMA method (Procedure for Uncertainty Management). In accordance with the standards, methods for measurement evaluation, which are based on Gum, are compiled within this standard. These also constitute the basis for the subsequent mathematical representation $[4,5]$.

\subsubsection{Mathematical process}

According to the afore mentioned standards, the evaluation of a measurement including the assessment of measurement uncertainty can be conducted in difference steps: (The approach compliant with GUM is subdivided into eight steps)

- Establishment of a model, which describes the measurement values' connection to all other involved values mathematically.

- Calculation of the measurement result and the measurement uncertainty for the measurement variable from the prepared data.

- Specification of the complete measurement result and determination of further uncertainty. 
Basis for the assessment of measurement uncertainty is a model, which describes the connection between measurement value $\mathrm{y}$ as result variable and the input variables $x_{1}, x_{2}, \ldots, x_{n}$ in the form of a mathematical function:

$$
y=f\left(x_{1}, x_{2}, \ldots, x_{n}\right)
$$

Provided that the measured variable has been measured multiple times, the arithmetic average has to be calculated from the individual measurement results:

$\bar{x}$ from characteristic values:

$$
\bar{x}=\frac{1}{n} \sum_{i=1}^{n} x_{i}
$$

It denotes the unwarranted measurement result, which is directly available in a measurement. If several measurements are conducted, the standard deviation from the average $u$ has to be determined. It is calculated from the standard deviation of the attention value $s$, whereas $n$ denotes the quantity of attention values:

$s$ from characteristic values:

$$
s=\sqrt{\frac{1}{n-1} \sum_{i=1}^{n}\left(x_{i}-\bar{x}\right)^{2}}
$$

The influences that take effect on a measurement evoke systematic and random measurement deviations. The known systematic deviation is used to correct the measurement result.

The random and unknown systematic deviations account for the measurement uncertainty. Their influence has to be estimated, unless it has been considered by the repeated measurement [6]. Thereto, data from earlier measurements, manufacturer's data or knowledge on the behavior and the characteristics of measurement objects and measurement sensors can be used.

According to the standard the assessment of uncertainty percentage via repeated measurement is designated method $\mathrm{A}$ and the assessment via alternative means is designated method B [7]. It is of crucial importance for the subsequent roundup of all measurement uncertainty components that the individual components' employed scatter parameters are equivalent and therefore transmittable.

This is achieved through interpreting the measurement uncertainty as standard deviation. In order to obtain a complete measurement result, the correction has to be added. The result provides the best estimate for the measurement parameter, which is determined via this measurement. For the determination of measurement uncertainty via method $B$, the standard deviation as well as the standard uncertainty have to be calculated from the available information. Following the determination of standard uncertainty $u$ :

Method type A (Normal distribution): Statistical analysis (series of measurements)

$$
u=s
$$

Method type B (Rectangular distribution):

- Other information (Value is between a lower limit -a and an upper limit + a)

- All values in between are equally likely (no further information)

- Specification in a calibration certificate [8]

$$
\begin{gathered}
\bar{x}=\frac{\left(a_{+}+a_{-}\right)}{2} \\
u=\frac{\Delta a}{\sqrt{3}}
\end{gathered}
$$


In accordance with [1], determination B (method B) is based upon earlier measurements, experience, specifications in calibration certificates or in manufacturer catalogues.

Catalogue specifications are then interpreted as extended measurement uncertainty U. Typically, catalogue specifications do not list information on probability distribution and level of reliability.

In these cases, normal distribution and a reliability level of $95.45 \%$ are assumed and an amplification factor of $\mathrm{k}=2$ is set. Hereby, a measurement uncertainty specification from a catalogue can be ascribed to the standard uncertainty $u$ :

$$
u=\frac{U}{k}
$$

This becomes necessary in cases, in which several measurement uncertainty components $u_{1}, u_{2}, u_{3}, \ldots u_{n}$ are to be combined to standard uncertainty $u_{c}$ :

$$
u_{c}=\sqrt{u_{1}^{2}+u_{2}^{2}+u_{3}^{2}+\cdots+u_{n}^{2}}
$$

The extended measurement uncertainty $U$ is calculated from the combined standard uncertainty with the amplification factor $k$, whereby an amplification factor of $k=2$ is usually assumed for a reliability level of $95.45 \%$.

$$
U=k \cdot u_{c}
$$

\section{Experimental determination of avoidable influences from practice}

Avoidable influences must be prevented in a geometrical measurement's early phase and should not be considered in the calculation of a measurement uncertainty. The following study demonstrates a classic case within the industries, which is often predetermined as an inspection criterion in technical requirements and results in the misjudgement of a measurement uncertainty.

Circle measurements are among the most common measurement assignments in product development. In accordance with [9], operators often ask themselves, with how many measuring points a workpieces' circles or cylinders are to be measured. The difficulty in measuring the radii and the associated circle segment is, among others, the identification of the discharge radius, which is hard to identify on a tangible workpiece.

In accordance with [10] the radius of a circle can be prefixed in order to eradicate this problem.

When, for example, the circle is calculated from measurement points, the circle center and the circle diameter normally have to be calculated. As an alternative side condition, the diameter can be prefixed and the center has to be calculated.

In accordance with the standard, at least $90^{\circ}$ of a circle segment have to be existent as measureable contour to specify radii [11]. However, this is seldom arranged in the technical drawings. Although this provision (at least $90^{\circ}$ of a circle segment existent as measureable contour) applies to synthetic material, different circle segments are measured with visualand tactile sensors in the following experiment, which features the example of a calibrated testing pin (maximum permissible deviation of $\pm 2 \mu \mathrm{m}$ ), revealing fluctuation in metallic materials as well.

Table 1 and Figure 2 provide an overview of deviations using the example of a calibrated testing pin under the provision of different circle segments. 
Table 1. Evaluation on a calibrated Pin Gauge.

\begin{tabular}{|c|c|c|c|}
\hline \multicolumn{4}{|c|}{$\begin{array}{c}\text { Tested on a calibrated Pin Gauge } \\
\text { Reference Diameter = 10,000 } \mathbf{~ m m}\end{array}$} \\
\hline $\begin{array}{c}\text { Circular } \\
\text { Segment [ }\end{array}$ & $\begin{array}{c}\text { Scanpoint } \\
\text { O/T }\end{array}$ & $\begin{array}{c}\text { Optical } \\
\text { Radius } \\
\text { [mm] }\end{array}$ & $\begin{array}{c}\text { Tactile } \\
\text { Radius } \\
\text { [mm] }\end{array}$ \\
\hline $15^{\circ}$ & $8 / 12$ & 4,847 & 4,846 \\
\hline $30^{\circ}$ & $17 / 15$ & 4,890 & 4,892 \\
\hline $45^{\circ}$ & $36 / 31$ & 4,953 & 4,956 \\
\hline $60^{\circ}$ & $47 / 43$ & 4,965 & 4,963 \\
\hline $75^{\circ}$ & $64 / 61$ & 4,972 & 4,971 \\
\hline $90^{\circ}$ & $109 / 107$ & 4,995 & 4,996 \\
\hline $120^{\circ}$ & $132 / 124$ & 5,000 & 5,001 \\
\hline $150^{\circ}$ & $181 / 169$ & 5,001 & 5,001 \\
\hline $180^{\circ}$ & $234 / 219$ & 5,001 & 5,000 \\
\hline $270^{\circ}$ & $327 / 322$ & 5,000 & 5,001 \\
\hline $360^{\circ}$ & $378 / 367$ & 5,001 & 5,001 \\
\hline
\end{tabular}

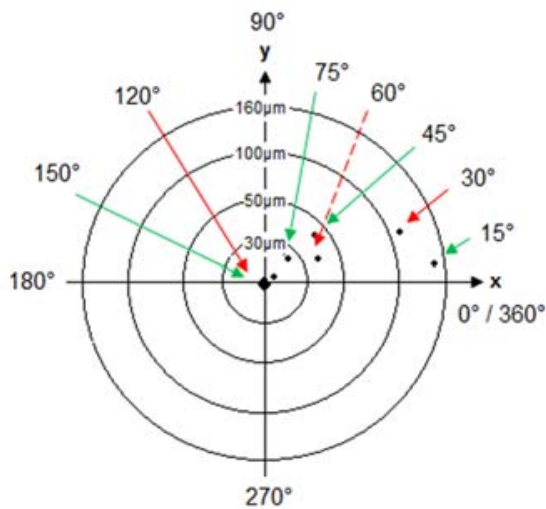

Fig. 2. Uncertainty to the reference middle point.

For stability reasons, measurements were repeated fivefold per circle segment following the Gauss-strategy. The result for the radius thus complies with the average of five measurements. In a first step, $15^{\circ}$ of the ideal contour were taken in and measured with the calibrated testing pin. Figure 2 reveals that the circle center shows a measurement deviation from the reference focus larger than $15 \mu \mathrm{m}$. It becomes clear that measurements under an available circle segment of $90^{\circ}$ reveal different or rather fluctuating deviations. Measurements over $90^{\circ}$ show comparable measurement values, merely an inevitable random measurement deviation comes into effect here. It is also important to note, that the measurement results become more stable with an increased number of recorded measuring points.

\section{Conclusions}

This article describes measurement deviations and measurement uncertainty with the help of a clear concept in detail. Influences which take an effect on measurement results and hence lead to measurement uncertainties were highlighted experimentally. The idea was to show that not all influences, which are known prior to an experiment, actually have a substantial effect on the measurement result. Furthermore, non-avoidable influences were described, which always occur under certain conditions and steadily afflict the measurement result with a measurement uncertainty. Through the establishment of a measurement uncertainty record, small influences can be analyzed and further avoided. Future research should focus on the observation of measurement uncertainties in workpieces with alternating properties, such as color, surface etc.

\section{References}

1. W. Dutschke, Manufacturing Metrology, (Teubner Verlag, 2002)

2. ISO 1 (Standard), Reference temperature for geometric product specification (2016)

3. ISO 14253-2 (Standard), Guidance for the estimation of uncertainty (2011)

4. DIN 1319-3 (Standard), Evaluation of measurements of a single measurand (1996)

5. DIN 1319-4 (Standard), Evaluation of measurements (in German) (1999)

6. ISO 22514-7 (Standard), Capability of measurement processes (2012)

7. ISO/IEC 17025 (Standard), General requirements for calibration laboratories (2005)

8. T. Hausotte, Manufacturing Metrology (in German) (Erlangen University, 2015)

9. F. Cebulla, G. Smith, S. Midha, NMS (in German), QZ Hanser Verlag, 56 (2004)

10. A. Weckenmann, Coordinate measuring technique (in German) (Hanser Verlag, 2012)

11. DIN 16742 (Standard), Tolerances and acceptance conditions (2013) 